\title{
Evaluation of Newer Insecticide Combinations against Bollworm Complex in Cotton
}

\author{
Bhujade Dnyaneshwar Rambhau*, R. Zanwar Purushottam and \\ Bokan Sanjog Chandrakant
}

\begin{abstract}
Department of Agricultural Entomology, Vasantrao Naik Marathwada Krishi Vidyapeeth, Parbhani-431402 (Maharashtra), India
\end{abstract}

*Corresponding author

\section{A B S T R A C T}

\section{Keywords}

Cotton, Bollworm complex, Insecticide

combination,

Pectinophora

Article Info

Accepted:

26 June 2018

Available Online:

10 July 2018
The evaluation of newer insecticide combinations against bollworm complex in cotton were studied during kharif 2017-2018 was conducted on the farm of Department of Agricultural Entomology, Vasantrao Naik Marathwada Agriculture University, Parbhani. The results revealed that application of chlorantraniliprole $8.8 \%$ + thiamethoxam $17.5 \%$ SC proved effective in recording minimum green fruiting bodies damage as well as per cent shed material, which was at par with indoxacarb $14.5 \%$ + acetamiprid $7.7 \%$ SC, chlorantraniliprole $9.3 \%$ + lambda cyhalothrin $4.6 \%$ ZC Thiamethoxam $12.6 \%$ + Lambda cyhalothrin 9.5\% ZC, flubendiamide $19.92 \%$ + thiacloprid $19.92 \%$ SC, spinatorum $10 \%$ + sulfoxaflor $30 \% \mathrm{WG}$ and thiamethoxam $12.6 \%$ + lambda cvhalothrin $9.5 \% \mathrm{ZC}$.

\section{Introduction}

Cotton is a major fiber crop of global significance, cultivated in more than seventy countries in the world. Cotton crop is playing an important role in economic, political and social affairs of the world. Cotton belongs to the family "Malvaceae" and genus "Gossypium". Cotton crop as commercial commodity plays an important role in industrial activity of nation, in terms of both employment generation and foreign exchange, hence it is popularly known as "White Gold" and "Friendly Fiber".
Major cotton growing states are Maharashtra, Madhya Pradesh, Gujarat from Central Zone, Andhra Pradesh, Tamil Nadu, Karnataka from South Zone and Punjab, Haryana, Rajasthan from North Zone. In Maharashtra, cotton is grown over an area of 41.98 lakh hectares with total production 85 lakh bales and an average productivity is $344 \mathrm{~kg}$ per hectare (Anonymous, 2018).

The pest spectrum of cotton crop is quite complex comprising of several species of the insects. Bollworm complex viz., American bollworm (Helicoverpa armigera Hub.), 
spotted bollworm (Earias vitella Fab.) and pink bollworm (Pectinophora gossypiella Saunders) account for a considerable yield loss to the extent of 36.2 per cent (Kranti et al., 2005).

A wide range of insecticides have proved as effective weapons in reducing the pest population. The cotton growers in India depend heavily on synthetic pesticides to combat pests and the crop consumes about 20 per cent of the total insecticides used in the country. However, the indiscriminate use of organophosphates, carbamates and synthetic pyrethroids has created a number of problems such as resistance and pest resurgence (Bajya et al., 2010). Hence the combination of insecticides was selected for reducing the bollworm population and avoids the insecticide resistance.

\section{Materials and Methods}

The experiment was conducted in randomized block design with nine treatments and three replications. The plot size of each treatment was $5.4 \mathrm{~m} \mathrm{X} 2.7 \mathrm{~m}$. NH-615 (non- $B t$ ) variety was used for experiment with spacing $60 \mathrm{~cm} \mathrm{X}$ $30 \mathrm{~cm}$.

\section{Effect of insecticides on infestation of bollworm in fruiting bodies}

The observation of infested squares, flowers, buds, bolls from each of observation plant from seven and fourteen days after each spraying was taken. The per cent damage in fruiting bodies was worked out by using following formula.

$\%$ damage in green fruiting bodies $=$

Damaged green fruiting bodies

Total green fruiting bodies
Effect of insecticides on infestation of bollworm in shed material

The shed squares, flowers, buds, bolls from each of observation plant were collected seven and fourteen days after each spraying. The shed fruiting bodies was collected in plastic bags and taken to the laboratory for the differentiation between damaged and undamaged bodies based on bollworm infestation. The per cent damage in shed material was worked out by using following formula.

$\%$ Shed material $=$

Shed material after insecticide treatment X 100

Total shed material (PTC)

\section{Effect of insecticide on locule damage}

At each picking number of locules from each plot was counted and sorted out into infested and healthy ones and percentage of infestation will be worked out.

\section{Effect of insecticides on yield of seed cotton}

The three pickings of seed cotton were done and yield of seed from each plot will be worked out in quintale per hectare basis.

\section{Results and Discussion}

Effect of insecticides on infestation of bollworm in fruiting bodies

The observations recorded 7 Days and 14 Days after each spraying i.e., first spray, second spray and third spray showed significant treatment differences in per cent shed material due to bollworm damage.

The mean data (Table 1) indicated that all treatments are significantly effective than control in reducing per cent fruiting body. The 
treatment chlorantraniliprole $8.8 \%+$ thiamethoxam $17.5 \% \mathrm{SC}$ recorded minimum per cent fruiting body damage $(5.00 \%)$ which was at par with indoxacarb $14.5 \%+$ acetamiprid $7.7 \quad \% \quad \mathrm{SC} \quad(5.52 \%)$, chlorantraniliprole $9.3 \%$ + lambda cyhalothrin $4.6 \%$ ZC $(6.05 \%)$, flubendiamide $19.92 \%+$ thiacloprid $19.92 \% \mathrm{SC} \quad(6.35 \%)$ spinatorum $10 \%$ + sulfoxaflor $30 \%$ (6.65\%). The next best treatments was observed that thiamethoxam $12.6 \%$ + lambda cyhalothrin $9.5 \% \mathrm{ZC}$ (6.97\%), novaluron $5.25 \%+$ indoxacarb $4.5 \%$ SC (7.50\%), profenophos $40 \%$ + cypermethrin $4 \%$ EC (7.94\%), cypermethrin $3 \%+$ quinalphos $20 \% \mathrm{EC}(13.59 \%)$.

The present findings are more or less parallel to Borude et al., (2018) studied the comparative efficacy of ready mix insecticides against bollworm complex of cotton. The results revealed that application of indoxacarb $14.5 \%+$ acetamiprid $7.7 \%$ SC proved effective in recording minimum green fruiting bodies damage, which was closely followed by profenofos $40 \%$ + cypermethrin $4 \%$ EC, novaluron $5.25 \%+$ indoxacarb $4.5 \% \mathrm{SC}$ and thiamethoxam $12.6 \%$ + lambda cyhalothrin $9.5 \% \mathrm{ZC}$.

\section{Effect of insecticides on infestation of bollworm in shed material}

The observations recorded 7 Days and 14 Days after each spraying i.e first spray, second spray and third spray showed significant treatment differences in per cent shed material due to bollworm damage.

The overall mean of three sprays (Table 2) revealed that the per cent shed material was ranged between 4.14 to 15.01 indicated that all treatments are significantly effective than control in reducing per cent shed material due to bollworm damage. Among them, chlorantraniliprole $8.8 \%+$ thiamethoxam $17.5 \%$ SC minimum per cent shed material $(4.14 \%)$ was statistically at par with indoxacarb $14.5 \%$ + acetamiprid $7.7 \%$ SC (4.72\%), chlorantraniliprole $9.3 \%$ + lambda cyhalothrin $4.6 \%$ ZC (5.02\%), flubendiamide $19.92 \%$ + thiacloprid $19.92 \% \mathrm{SC}(5.32 \%)$, spinatorum $10 \%$ + sulfoxaflor $30 \%$ (5.28\%) and thiamethoxam $12.6 \%+$ lambda cyhalothrin $9.5 \%$ ZC (5.42\%). Follwoed by novaluron $5.25 \%+$ indoxacarb $4.5 \% \mathrm{SC}$ (6.44\%), profenophos $40 \%+$ cypermethrin $4 \%$ EC $(7.55 \%)$, cypermethrin $3 \%+$ quinolphos $20 \%$ EC (9.56\%).

The present findings were more or less parallel to Borude et al., (2018) studied the comparative efficacy of ready mix insecticides against bollworm complex of cotton. The results revealed that application of indoxacarb $14.5 \%+$ acetamiprid $7.7 \%$ SC proved effective in recording minimum green fruiting bodies damage, which was closely followed by profenofos $40 \%$ + cypermethrin $4 \% \mathrm{EC}$, novaluron $5.25 \%$ + indoxacarb $4.5 \% \mathrm{SC}$ and thiamethoxam $12.6 \%$ + lambda cyhalothrin 9.5\% ZC. Also, Bajya et al., (2015) observed that Ampligo $150 \mathrm{ZC}$ (combination of chlorantranilipole $9.3 \%$ + lambdacyhalothrin $4.6 \%$ ZC) was highly effective in checking the larval population of $H$. armigera compared to standard check Ampligo 150 ZC (30 g a.i. /ha), chlorantranilipole 18.5 SC @ 30g a.i. /ha, qunalphos 25 EC @ $500 \mathrm{~g}$ a.i. /ha, deltamethrin 2.8 EC @ $12.5 \mathrm{~g}$ a.i. /ha and lambda cyhalothrin 4.9 CS @ $25 \mathrm{~g}$ a.i./ha.

The data displayed in Table 3 indicated that all the treatments are significantly superior over control in lowering the per cent locale damage. Among them minimum locule damage $(35.29 \%)$ is observed in the plot sprayed with chlorantraniliprole $8.8 \%+$ thiamethoxam $17.5 \% \mathrm{SC}$ and this treatment was statistically at par with indoxacarb $14.5 \%$ + acetamiprid $7.7 \%$ SC (38.81\%), chlorantraniliprole $9.3 \%$ + lambda cyhalothrin 4.6\% ZC (44.29\%), flubendiamide $19.92 \%+$ thiacloprid $19.92 \% \mathrm{SC} \quad(46.38 \%)$ and spinatorum $10 \%$ + sulfoxaflor $30 \%(57.98 \%)$. 
Table.1 Effect of different insecticides against fruiting body damage due to bollworm complex

\begin{tabular}{|c|c|c|c|c|c|c|c|c|c|c|c|}
\hline \multirow{2}{*}{$\begin{array}{l}\text { Tr. } \\
\text { No }\end{array}$} & \multirow[t]{2}{*}{ Treatment } & \multirow{2}{*}{$\begin{array}{l}\text { Dose } \\
\text { a.i/ha }\end{array}$} & \multirow{2}{*}{$\begin{array}{c}\text { Conc. } \\
\%\end{array}$} & \multicolumn{3}{|c|}{ First Spray } & \multicolumn{2}{|c|}{ Second Spray } & \multicolumn{2}{|c|}{ Third Spray } & \multirow[t]{2}{*}{ Mean } \\
\hline & & & & PTC & $\begin{array}{c}7 \\
\text { DAS }\end{array}$ & $\begin{array}{c}14 \\
\text { DAS }\end{array}$ & $\begin{array}{c}7 \\
\text { DAS }\end{array}$ & $\begin{array}{c}14 \\
\text { DAS }\end{array}$ & $\begin{array}{c}7 \\
\text { DAS }\end{array}$ & $\begin{array}{c}14 \\
\text { DAS }\end{array}$ & \\
\hline 1 & $\begin{array}{l}\text { Chlorantraniliprole } 9.3 \% \text { + Lambda } \\
\text { cyhalothrin } 4.6 \% \mathrm{ZC}\end{array}$ & $37.5 \mathrm{~g}$ & $0.006 \%$ & $\begin{array}{c}12.38 \\
(20.37)\end{array}$ & $\begin{array}{c}4.88 \\
(12.51)\end{array}$ & $\begin{array}{c}6.50 \\
(14.74)\end{array}$ & $\begin{array}{c}5.82 \\
(13.91)\end{array}$ & $\begin{array}{c}6.89 \\
(15.19)\end{array}$ & $\begin{array}{c}5.44 \\
(13.31)\end{array}$ & $\begin{array}{c}6.79 \\
(15.10)\end{array}$ & $\begin{array}{c}6.05 \\
(14.20)\end{array}$ \\
\hline 2 & $\begin{array}{l}\text { Flubendiamide } 19.92 \% \text { + Thiacloprid } \\
19.92 \% \text { SC }\end{array}$ & $\begin{array}{c}48+48 \\
g\end{array}$ & $0.02 \%$ & $\begin{array}{c}12.94 \\
(20.81)\end{array}$ & $\begin{array}{c}5.24 \\
(13.02)\end{array}$ & $\begin{array}{c}6.59 \\
(14.86)\end{array}$ & $\begin{array}{c}5.86 \\
(14.00)\end{array}$ & $\begin{array}{c}6.98 \\
(15.31)\end{array}$ & $\begin{array}{c}6.42 \\
(14.65)\end{array}$ & $\begin{array}{c}7.02 \\
(15.35)\end{array}$ & $\begin{array}{c}6.35 \\
(14.58)\end{array}$ \\
\hline 3 & Profenophos $40 \%$ + Cypermethrine $4 \%$ EC & $440 \mathrm{~g}$ & $0.088 \%$ & $\begin{array}{c}10.29 \\
(18.49)\end{array}$ & $\begin{array}{c}6.94 \\
(15.23)\end{array}$ & $\begin{array}{c}8.55 \\
(16.98)\end{array}$ & $\begin{array}{c}7.22 \\
(15.55)\end{array}$ & $\begin{array}{c}8.71 \\
(17.15)\end{array}$ & $\begin{array}{c}7.35 \\
(15.67)\end{array}$ & $\begin{array}{c}8.87 \\
(17.14)\end{array}$ & $\begin{array}{c}7.94 \\
(16.35)\end{array}$ \\
\hline 4 & Cypermethrin $3 \%$ + Quinalphos 20\% EC & $230 \mathrm{~g}$ & $0.046 \%$ & $\begin{array}{c}17.79 \\
(24.75)\end{array}$ & $\begin{array}{c}12.33 \\
(20.54)\end{array}$ & $\begin{array}{c}14.31 \\
(22.03)\end{array}$ & $\begin{array}{c}12.97 \\
(20.73)\end{array}$ & $\begin{array}{c}15.04 \\
(22.80)\end{array}$ & $\begin{array}{c}12.60 \\
(20.51)\end{array}$ & $\begin{array}{c}14.3 \\
(21.19)\end{array}$ & $\begin{array}{c}13.59 \\
(21.58)\end{array}$ \\
\hline 5 & Indoxacarb $14.5 \%$ + Acetamiprid $7.7 \% \mathrm{SC}$ & $88.8 \mathrm{~g}$ & $0.046 \%$ & $\begin{array}{c}12.99 \\
(21.07)\end{array}$ & $\begin{array}{c}4.67 \\
(12.42)\end{array}$ & $\begin{array}{c}6.09 \\
(14.22)\end{array}$ & $\begin{array}{c}5.23 \\
(13.08)\end{array}$ & $\begin{array}{c}6.00 \\
(14.11)\end{array}$ & $\begin{array}{c}5.06 \\
(12.53)\end{array}$ & $\begin{array}{c}6.07 \\
(13.24)\end{array}$ & $\begin{array}{c}5.52 \\
(13.57)\end{array}$ \\
\hline 6 & $\begin{array}{l}\text { Thiamethoxam } 12.6 \%+\text { Lambda } \\
\text { cyhalothrin } 9.5 \% \text { ZC }\end{array}$ & $44 \mathrm{~g}$ & $0.008 \%$ & $\begin{array}{c}11.94 \\
(20.05)\end{array}$ & $\begin{array}{c}5.70 \\
(13.70)\end{array}$ & $\begin{array}{c}7.19 \\
(15.42)\end{array}$ & $\begin{array}{c}6.81 \\
(14.93)\end{array}$ & $\begin{array}{c}7.86 \\
(16.23)\end{array}$ & $\begin{array}{c}7.01 \\
(14.98)\end{array}$ & $\begin{array}{c}7.27 \\
(15.47)\end{array}$ & $\begin{array}{c}6.97 \\
(15.18)\end{array}$ \\
\hline 7 & $\begin{array}{l}\text { Chlorantraniliprole } 8.8 \%+\text { Thiamethoxam } \\
17.5 \% \text { SC }\end{array}$ & $150 \mathrm{~g}$ & $0.026 \%$ & $\begin{array}{c}11.62 \\
(19.81)\end{array}$ & $\begin{array}{c}3.71 \\
(10.99)\end{array}$ & $\begin{array}{c}5.70 \\
(13.71)\end{array}$ & $\begin{array}{c}4.98 \\
(12.87)\end{array}$ & $\begin{array}{c}5.30 \\
(13.29)\end{array}$ & $\begin{array}{c}4.93 \\
(12.82)\end{array}$ & $\begin{array}{c}5.82 \\
(13.84)\end{array}$ & $\begin{array}{c}5 \\
(12.87)\end{array}$ \\
\hline 8 & Novaluron $5.25 \%$ + Indoxacarb $4.5 \% \mathrm{SC}$ & $\begin{array}{l}43.31+ \\
37.13 \mathrm{~g}\end{array}$ & $0.019 \%$ & $\begin{array}{l}11.87 \\
(20.12)\end{array}$ & $\begin{array}{c}6.54 \\
(14.57)\end{array}$ & $\begin{array}{c}7.61 \\
(15.79)\end{array}$ & $\begin{array}{c}7.07 \\
(15.39)\end{array}$ & $\begin{array}{c}8.68 \\
(16.84)\end{array}$ & $\begin{array}{c}7.12 \\
(15.28)\end{array}$ & $\begin{array}{c}8.36 \\
(16.67)\end{array}$ & $\begin{array}{c}7.5 \\
(15.95)\end{array}$ \\
\hline 9 & Spinatorum $10 \%+$ Sulfoxaflor $30 \%$ & $120 \mathrm{~g}$ & $0.002 \%$ & $\begin{array}{c}11.60 \\
(19.86)\end{array}$ & $\begin{array}{c}5.28 \\
(13.04)\end{array}$ & $\begin{array}{c}6.78 \\
(14.93)\end{array}$ & $\begin{array}{c}6.18 \\
(14.12)\end{array}$ & $\begin{array}{c}7.55 \\
(15.84)\end{array}$ & $\begin{array}{c}7.00 \\
(15.22)\end{array}$ & $\begin{array}{c}7.12 \\
(15.47)\end{array}$ & $\begin{array}{c}6.65 \\
(14.93)\end{array}$ \\
\hline 10 & Untreated control & - & - & $\begin{array}{c}12.51 \\
(20.68)\end{array}$ & $\begin{array}{c}30.9 \\
(33.74)\end{array}$ & $\begin{array}{c}48.85 \\
(44.32)\end{array}$ & $\begin{array}{c}72.14 \\
(58.12)\end{array}$ & $\begin{array}{c}84.73 \\
(66.97)\end{array}$ & $\begin{array}{c}91.72 \\
(73.29)\end{array}$ & $\begin{array}{c}96.12 \\
(78.61)\end{array}$ & $\begin{array}{c}70.74 \\
(57.23)\end{array}$ \\
\hline & $\mathrm{SE} \pm$ & & & - & 1.38 & 1.34 & 1.38 & 1.06 & 1.45 & 1.70 & 0.59 \\
\hline & CD@0.5\% & & & NS & 4.15 & 4.03 & 4.14 & 3.18 & 4.35 & 5.10 & 1.78 \\
\hline & $\mathrm{CV} \%$ & & & - & 15.04 & 12.46 & 12.45 & 8.61 & 12.09 & 13.25 & 5.26 \\
\hline
\end{tabular}

Figure in parentheses are angular transformed values. PTC- Pre treatment count

DAS- Days after spraying 
Table.2 Effect of different insecticides on per cent shed material due to bollworm damage

\begin{tabular}{|c|c|c|c|c|c|c|c|c|c|c|c|}
\hline \multirow[t]{2}{*}{ Tr.No } & \multirow[t]{2}{*}{ Treatment } & \multirow{2}{*}{$\begin{array}{l}\text { Dose } \\
\text { a.i/ha }\end{array}$} & \multirow{2}{*}{$\begin{array}{c}\text { Conc. } \\
\%\end{array}$} & \multicolumn{3}{|c|}{ First Spray } & \multicolumn{2}{|c|}{ Second Spray } & \multicolumn{2}{|c|}{ Third Spray } & \multirow[t]{2}{*}{ Mean } \\
\hline & & & & PTC & $\begin{array}{c}7 \\
\text { DAS }\end{array}$ & $\begin{array}{c}14 \\
\text { DAS }\end{array}$ & $\begin{array}{c}7 \\
\text { DAS }\end{array}$ & $\begin{array}{c}14 \\
\text { DAS }\end{array}$ & $\begin{array}{c}7 \\
\text { DAS }\end{array}$ & $\begin{array}{c}14 \\
\text { DAS }\end{array}$ & \\
\hline 1 & $\begin{array}{l}\text { Chlorantraniliprole } 9.3 \%+ \\
\text { Lambda cyhalothrin } 4.6 \% \mathrm{ZC}\end{array}$ & $37.5 \mathrm{~g}$ & $\begin{array}{c}0.006 \\
\%\end{array}$ & $\begin{array}{c}9.42 \\
(17.77)\end{array}$ & $\begin{array}{c}3.98 \\
(11.49)\end{array}$ & $\begin{array}{c}5.30 \\
(13.23)\end{array}$ & $\begin{array}{c}4.52 \\
(12.12)\end{array}$ & $\begin{array}{c}5.97 \\
(14.07)\end{array}$ & $\begin{array}{c}4.71 \\
(12.94)\end{array}$ & $\begin{array}{c}5.66 \\
(13.71)\end{array}$ & $\begin{array}{c}5.02 \\
(12.93)\end{array}$ \\
\hline 2 & $\begin{array}{l}\text { Flubendiamide } 19.92 \%+ \\
\text { Thiacloprid } 19.92 \% \text { SC }\end{array}$ & $\begin{array}{c}48+48 \\
g\end{array}$ & $0.02 \%$ & $\begin{array}{c}13.02 \\
(21.13)\end{array}$ & $\begin{array}{c}4.01 \\
(11.54)\end{array}$ & $\begin{array}{c}5.42 \\
(13.39)\end{array}$ & $\begin{array}{c}4.69 \\
(12.45)\end{array}$ & $\begin{array}{c}6.09 \\
(14.19)\end{array}$ & $\begin{array}{c}5.62 \\
(13.64)\end{array}$ & $\begin{array}{c}6.11 \\
(14.17)\end{array}$ & $\begin{array}{c}5.32 \\
(13.32)\end{array}$ \\
\hline 3 & $\begin{array}{l}\text { Profenophos } 40 \% \text { + Cypermethrin } \\
4 \% \text { EC }\end{array}$ & $440 \mathrm{~g}$ & $\begin{array}{c}0.088 \\
\%\end{array}$ & $\begin{array}{c}9.86 \\
(18.09)\end{array}$ & $\begin{array}{c}5.80 \\
(13.93)\end{array}$ & $\begin{array}{c}7.90 \\
(16.27)\end{array}$ & $\begin{array}{c}6.63 \\
(14.90)\end{array}$ & $\begin{array}{c}8.94 \\
(17.37)\end{array}$ & $\begin{array}{c}7.08 \\
(15.40)\end{array}$ & $\begin{array}{c}8.99 \\
(17.43)\end{array}$ & $\begin{array}{c}7.55 \\
(15.93)\end{array}$ \\
\hline 4 & $\begin{array}{l}\text { Cypermethrin } 3 \%+\text { Quinalphos } \\
20 \% \text { EC }\end{array}$ & $230 \mathrm{~g}$ & $\begin{array}{c}0.046 \\
\%\end{array}$ & $\begin{array}{c}13.76 \\
(21.53)\end{array}$ & $\begin{array}{c}8.97 \\
(17.39)\end{array}$ & $\begin{array}{c}10.49 \\
(18.89)\end{array}$ & $\begin{array}{c}8.91 \\
(17.30)\end{array}$ & $\begin{array}{c}10.70 \\
(19.01)\end{array}$ & $\begin{array}{c}8.59 \\
(16.88)\end{array}$ & $\begin{array}{c}9.97 \\
(18.24)\end{array}$ & $\begin{array}{c}9.65 \\
(18.03)\end{array}$ \\
\hline 5 & $\begin{array}{l}\text { Indoxacarb } 14.5 \%+\text { Acetamiprid } \\
7.7 \% \mathrm{SC}\end{array}$ & $88.8 \mathrm{~g}$ & $\begin{array}{c}0.046 \\
\%\end{array}$ & $\begin{array}{c}9.25 \\
(17.63)\end{array}$ & $\begin{array}{c}3.91 \\
(10.88)\end{array}$ & $\begin{array}{c}4.64 \\
(12.37)\end{array}$ & $\begin{array}{c}4.16 \\
(11.73)\end{array}$ & $\begin{array}{c}5.51 \\
(13.53)\end{array}$ & $\begin{array}{c}4.56 \\
(12.28)\end{array}$ & $\begin{array}{c}5.58 \\
(13.65)\end{array}$ & $\begin{array}{c}4.72 \\
(12.54)\end{array}$ \\
\hline 6 & $\begin{array}{l}\text { Thiamethoxam } 12.6 \%+\text { Lambda } \\
\text { cyhalothrin } 9.5 \% \mathrm{ZC}\end{array}$ & $44 \mathrm{~g}$ & $\begin{array}{c}0.008 \\
\%\end{array}$ & $\begin{array}{c}8.60 \\
(16.93)\end{array}$ & $\begin{array}{c}4.11 \\
(11.69)\end{array}$ & $\begin{array}{c}5.63 \\
(13.63)\end{array}$ & $\begin{array}{c}5.23 \\
(13.03)\end{array}$ & $\begin{array}{c}6.23 \\
(14.41)\end{array}$ & $\begin{array}{c}4.88 \\
(12.59)\end{array}$ & $\begin{array}{c}6.45 \\
(14.58)\end{array}$ & $\begin{array}{c}5.42 \\
(13.44)\end{array}$ \\
\hline 7 & $\begin{array}{l}\text { Chlorantraniliprole } 8.8 \%+ \\
\text { Thiamethoxam } 17.5 \% \mathrm{SC}\end{array}$ & $150 \mathrm{~g}$ & $\begin{array}{c}0.026 \\
\%\end{array}$ & $\begin{array}{c}8.90 \\
(17.32)\end{array}$ & $\begin{array}{c}2.87 \\
(9.27)\end{array}$ & $\begin{array}{c}4.46 \\
(12.17)\end{array}$ & $\begin{array}{c}3.31 \\
(10.48)\end{array}$ & $\begin{array}{c}4.99 \\
(12.90)\end{array}$ & $\begin{array}{c}3.80 \\
(11.22)\end{array}$ & $\begin{array}{c}5.44 \\
(13.48)\end{array}$ & $\begin{array}{c}4.14 \\
(11.73)\end{array}$ \\
\hline 8 & $\begin{array}{l}\text { Novaluron } 5.25 \%+\text { Indoxacarb } \\
4.5 \% \mathrm{SC}\end{array}$ & $\begin{array}{l}43.31+ \\
37.13 \mathrm{~g}\end{array}$ & $\begin{array}{c}0.019 \\
\%\end{array}$ & $\begin{array}{c}8.62 \\
(16.93)\end{array}$ & $\begin{array}{c}5.47 \\
(13.51)\end{array}$ & $\begin{array}{c}7.86 \\
(16.27)\end{array}$ & $\begin{array}{c}6.05 \\
(14.12)\end{array}$ & $\begin{array}{c}6.52 \\
(14.61)\end{array}$ & $\begin{array}{c}5.11 \\
(12.95)\end{array}$ & $\begin{array}{c}7.67 \\
(16.01)\end{array}$ & $\begin{array}{c}6.44 \\
(14.70)\end{array}$ \\
\hline 9 & $\begin{array}{l}\text { Spinatorum } 10 \%+\text { Sulfoxaflor } \\
30 \%\end{array}$ & $120 \mathrm{~g}$ & $\begin{array}{c}0.002 \\
\%\end{array}$ & $\begin{array}{c}8.37 \\
(16.79)\end{array}$ & $\begin{array}{c}4.07 \\
(11.62)\end{array}$ & $\begin{array}{c}5.58 \\
(13.60)\end{array}$ & $\begin{array}{c}4.72 \\
(12.50)\end{array}$ & $\begin{array}{c}6.15 \\
(14.29)\end{array}$ & $\begin{array}{c}4.84 \\
(12.59)\end{array}$ & $\begin{array}{c}6.36 \\
(14.46)\end{array}$ & $\begin{array}{c}5.28 \\
(13.11)\end{array}$ \\
\hline 10 & Untreated control & - & - & $\begin{array}{c}10.05 \\
(17.82)\end{array}$ & $\begin{array}{c}14.15 \\
(21.60)\end{array}$ & $\begin{array}{c}16.04 \\
(23.16)\end{array}$ & $\begin{array}{c}14.66 \\
(23.53\end{array}$ & $\begin{array}{c}16.00 \\
(23.53)\end{array}$ & $\begin{array}{l}13.88 \\
(21.71)\end{array}$ & $\begin{array}{c}15.38 \\
(22.93)\end{array}$ & $\begin{array}{c}15.01 \\
(22.77)\end{array}$ \\
\hline & $\mathrm{SE} \pm$ & & & - & 1.27 & 1.45 & 0.86 & 0.93 & 1.16 & 1.14 & 0.58 \\
\hline & CD@0.5\% & & & NS & 3.80 & 4.35 & 2.58 & 1.32 & 3.47 & 3.41 & 1.74 \\
\hline & $\mathrm{CV} \%$ & & & - & 16.49 & 16.44 & 10.50 & 10.27 & 14.16 & 12.46 & 6.77 \\
\hline
\end{tabular}

Figure in parentheses are angular transformed values.

PTC- Pre treatment count

DAS- Days after spraying 
Table.3 Effect of newer insecticides on the locule damage, per cent bad kapas, per cent seed damage and yield per ha.

\begin{tabular}{|c|c|c|c|c|c|c|c|}
\hline $\begin{array}{l}\text { Tr. } \\
\text { No. }\end{array}$ & Treatment & $\begin{array}{l}\text { Dose } \\
\text { a.i / ha }\end{array}$ & Conc $\%$ & $\begin{array}{l}(\%) \text { Locule } \\
\text { damage }\end{array}$ & $\begin{array}{l}\% \text { bad } \\
\text { kapas }\end{array}$ & $\begin{array}{l}\% \text { Seed } \\
\text { Damage }\end{array}$ & $\begin{array}{l}\text { Yield } \\
\text { qt/ha }\end{array}$ \\
\hline $\mathbf{T}_{1}$ & $\begin{array}{l}\text { Chlorantraniliprole } 9.3 \% \text { + Lambda } \\
\text { cyhalothrin } 4.6 \% \mathrm{ZC}\end{array}$ & $37.5 \mathrm{~g}$ & $0.006 \%$ & $\begin{array}{c}44.29 \\
(38.01)\end{array}$ & $\begin{array}{c}16.3 \\
(23.80)\end{array}$ & $\begin{array}{l}16.33 \\
(7.33)\end{array}$ & $\begin{array}{c}5.45 \\
(13.45)\end{array}$ \\
\hline $\mathbf{T}_{2}$ & $\begin{array}{l}\text { Flubendiamide } 19.92 \%+\text { Thiacloprid } \\
19.92 \% \mathrm{SC}\end{array}$ & $48+48 g$ & $0.02 \%$ & $\begin{array}{c}46.38 \\
(39.51)\end{array}$ & $\begin{array}{c}18.44 \\
(25.41)\end{array}$ & $\begin{array}{l}23.33 \\
(8.74)\end{array}$ & $\begin{array}{c}5.20 \\
(13.13)\end{array}$ \\
\hline $\mathbf{T}_{\mathbf{3}}$ & Profenophos $40 \%+$ Cypermethrine $4 \%$ EC & $440 \mathrm{~g}$ & $0.088 \%$ & $\begin{array}{c}72.28 \\
(55.98)\end{array}$ & $\begin{array}{c}30.06 \\
(33.43)\end{array}$ & $\begin{array}{c}46.66 \\
(12.45)\end{array}$ & $\begin{array}{c}3.11 \\
(10.15)\end{array}$ \\
\hline $\mathbf{T}_{4}$ & Cypermethrin $3 \%+$ Quinalphos $20 \%$ EC & $230 \mathrm{~g}$ & $0.046 \%$ & $\begin{array}{c}84.03 \\
(56.21)\end{array}$ & $\begin{array}{c}39.94 \\
(39.18)\end{array}$ & $\begin{array}{c}56.66 \\
(13.75)\end{array}$ & $\begin{array}{c}2.84 \\
(9.68)\end{array}$ \\
\hline $\mathbf{T}_{5}$ & $\begin{array}{l}\text { Indoxacarb } 14.5 \%+\text { Acetamiprid } 7.7 \% \\
\text { SC }\end{array}$ & $88.8 \mathrm{~g}$ & $0.046 \%$ & $\begin{array}{c}38.81 \\
(35.32)\end{array}$ & $\begin{array}{c}9.83 \\
(18.25)\end{array}$ & $\begin{array}{l}13.33 \\
(6.53)\end{array}$ & $\begin{array}{c}6.98 \\
(15.28)\end{array}$ \\
\hline $\mathbf{T}_{6}$ & $\begin{array}{l}\text { Thiamethoxam } 12.6 \%+\text { Lambda } \\
\text { cyhalothrin } 9.5 \% \mathrm{ZC}\end{array}$ & $44 \mathrm{~g}$ & $0.008 \%$ & $\begin{array}{c}63.86 \\
(45.36)\end{array}$ & $\begin{array}{c}25.53 \\
(29.00)\end{array}$ & $\begin{array}{c}30.0 \\
(9.87)\end{array}$ & $\begin{array}{c}4.49 \\
(12.21)\end{array}$ \\
\hline $\mathbf{T}_{7}$ & $\begin{array}{l}\text { Chlorantraniliprole } 8.8 \%+\text { Thiamethoxam } \\
17.5 \% \text { SC }\end{array}$ & $150 \mathrm{~g}$ & $0.026 \%$ & $\begin{array}{l}35.29 \\
(33.60)\end{array}$ & $\begin{array}{c}7.8 \\
(16.18)\end{array}$ & $\begin{array}{c}6.66 \\
(3.82)\end{array}$ & $\begin{array}{c}13.12 \\
(21.22)\end{array}$ \\
\hline $\mathbf{T}_{8}$ & Novaluron $5.25 \%$ + Indoxacarb $4.5 \%$ SC & $\begin{array}{c}43.31+37.13 \\
\mathrm{~g}\end{array}$ & $0.019 \%$ & $\begin{array}{c}70.73 \\
(49.74)\end{array}$ & $\begin{array}{c}27.67 \\
(31.60)\end{array}$ & $\begin{array}{c}36.66 \\
(11.02)\end{array}$ & $\begin{array}{c}3.64 \\
(10.99)\end{array}$ \\
\hline $\mathbf{T}_{9}$ & Spinatorum $10 \%+$ Sulfoxaflor $30 \%$ & $120 \mathrm{~g}$ & $0.002 \%$ & $\begin{array}{c}57.98 \\
(42.68)\end{array}$ & $\begin{array}{c}20.88 \\
(27.17)\end{array}$ & $\begin{array}{l}26.66 \\
(9.35)\end{array}$ & $\begin{array}{c}4.64 \\
(12.41)\end{array}$ \\
\hline \multirow[t]{4}{*}{$\mathbf{T}_{10}$} & Untreated control & - & - & $\begin{array}{c}91.73 \\
(62.36)\end{array}$ & $\begin{array}{c}88.84 \\
(70.45)\end{array}$ & $\begin{array}{c}63.33 \\
(14.56)\end{array}$ & $\begin{array}{c}1.62 \\
(7.30)\end{array}$ \\
\hline & $\mathrm{SE} \pm$ & & & 3.94 & 0.92 & 0.86 & 0.47 \\
\hline & CD@ $0.05 \%$ & & & 11.91 & 2.77 & 2.58 & 1.43 \\
\hline & $\mathrm{CV} \%$ & & & 15.49 & 5.10 & 15.34 & 6.59 \\
\hline
\end{tabular}

${ }^{*}$ values are angular transformed. 
The next best treatments were thiamethoxam $12.6 \%$ + lambda cyhalothrin $9.5 \%$ ZC $(63.86 \%)$, novaluron $5.25 \%+$ indoxacarb $4.5 \%$ SC $(70.73 \%)$ and profenophos $40 \%+$ cypermethrin $4 \%$ EC (72.28\%). Bajya et al., (2015) observed that Ampligo 150 ZC (combination of chlorantranilipole $9.3 \%+$ lambda cyhalothrin $4.6 \%$ ZC) was highly effective insignificant reduction of per cent damage on squares, bolls and locule during Kharif 2011 and 2013.

It is evident from Table 3 that all the treatments are significantly effective as compared to control in reducing per cent bad kapas and per cent seed damage due to bollworm. The treatment with chlorantraniliprole $8.8 \%+$ thiamethoxam $17.5 \%$ SC showed minimum per cent of bad kapas and per cent seed damage $(7.8 \%$ and $6.66 \%$ ) which was statistically at par with indoxacarb $14.5 \%$ + acetamiprid $7.7 \%$ SC $(9.83 \%$ and $13.33 \%)$.In other treatments viz., chlorantraniliprole $9.3 \%$ + lambda cyhalothrin $4.6 \% \mathrm{ZC}$ ( $16.3 \%$ and $16.33 \%)$, flubendiamide $19.92 \%$ + thiacloprid $19.92 \%$ SC $(18.44 \%$ and $23.33 \%$ ), spinatorum $10 \%$ + sulfoxaflor $30 \%$ (20.88\% and 26.66\%), thiamethoxam $12.6 \%$ + lambda cyhalothrin $9.5 \%$ ZC $(25.53 \%$ and $30.0 \%$ ), novaluron $5.25 \%+$ indoxacarb $4.5 \%$ SC (27.67\% and 36.66\%),profenophos $40 \%+$ cypermethrin $4 \%$ EC (30.06\% and $46.66 \%)$ and cypermethrin $3 \%+$ quinalphos $20 \% \mathrm{EC}$ (39.94\% and $56.66 \%)$ recorded minimum per cent bad kapas than untreated control. Bajya et al., (2015) as they reported that Ampligo $150 \mathrm{ZC}$ (combination of chlorantranilipole 9.3 $\%$ + lambda cyhalothrin $4.6 \%$ ZC) in cotton gave significant reduction of per cent damage on squares, bolls and loculi as well as high yield during Kharif 2011 and 2013 when compared to standard check Ampligo $150 \mathrm{ZC}$ (30 g a.i./ha), chlorantranilipole 18.5 SC @ $30 \mathrm{~g}$ a.i./ha, qunalphos $25 \mathrm{EC}$ @ $500 \mathrm{~g}$ a.i./ha, and lambda cyhalothrin 4.9 CS @ $25 \mathrm{~g}$ a.i./ha. The data on yield of seed cotton was recorded and presented in Table 3 reported that all the insecticides were found to be significantly superior in recording higher seed cotton yield over untreated control. The significantly higher yield of seed cotton yield (13.12 q/ha) was recorded in the treatment with chlorantraniliprole $8.8 \%+$ thiamethoxam $17.5 \%$ SC. Followed by treatments were indoxacarb $14.5 \%$ + acetamiprid $7.7 \%$ SC (6.98 q/ha), chlorantraniliprole $9.3 \%+$ lambda cyhalothrin $4.6 \%$ ZC (5.45 q/ha), stood $3^{\text {rd }}$ and it was at par with flubendiamide $19.92 \%$ + thiacloprid $19.92 \%$ SC (5.20), spinatorum $10 \%+$ sulfoxaflor $30 \%$ (4.64 $\mathrm{q} / \mathrm{ha}$ ) and thiamethoxam $12.6 \%+$ lambda cyhalothrin $9.5 \%$ ZC (4.49 q/ha), novaluron $5.25 \%$ + indoxacarb $4.5 \%$ SC (3.64 q/ha) being next effective treatment and at par with profenophos $40 \%$ + cypermethrin $4 \%$ EC (3.11 q/ha) and cypermethrin $3 \%+$ quinalphos $20 \%$ EC (2.84 q/ha). Bajya et al., (2015) who reported that Ampligo 150 ZC (combination of chlorantranilipole $9.3 \%+$ lambda cyhalothrin $4.6 \% \mathrm{ZC}$ ) in cotton gave significant reduction of per cent damage on squares, bolls and loculi as well as high yield during Kharif 2011 and 2013.

\section{References}

Anonymous (2018). Annual report of cotton corporation of India.10-32.

Bajya D.R., Baheti H.S. and Raza S.K. (2015). Field efficacy of newer insecticide formulation Ampligo 150 ZC against bollworm complex in cotton. J. Cotton Res. Dev. 29(1): 9498.

Bajya D.R., Monga D. and Tyagi M.P. (2010). Seasonal monitoring of Insecticide resistance in Helicoverpa armigera on cotton and chickpea. Indian J. Plant Prot. 38: 41-46.

Borude, B. S., Bhalkare, S.K., Undirwade, D. B., and Rathod, P. K. Ready mix insecticides for cotton bollworm 
complex.

Kranthi, K. R., Naidu, S., Dhawad, C. S., Tatwawadi, A., Mate, K., Patil, E., Bharose, A. A., Behere, G.T., Wadaskar, R.M and Kranthi, S. (2005). Temporal and intra-plant variability of Cry1Ac expression in $B t$-cotton and its influence (Hubner) (Noctuidae: Lepidoptera). Current science (2005): 291-298.

\section{How to cite this article:}

Bhujade Dnyaneshwar Rambhau, R. Zanwar Purushottam and Bokan Sanjog Chandrakant. 2018. Evaluation of Newer Insecticide Combinations against Bollworm Complex in Cotton. Int.J.Curr.Microbiol.App.Sci. 7(07): 3619-3626. doi: https://doi.org/10.20546/ijcmas.2018.707.421 